\title{
Comparative Study of Switched Reluctance Motors Performances for Two Current Distributions and Excitation Modes
}

\author{
G.J. $\mathrm{LI}^{1}$, X. OJEDA ${ }^{1}$, Member, IEEE, S. HLIOUI ${ }^{1}$, Member, IEEE, E. HOANG ${ }^{1}$, M. GABSI ${ }^{1}$ and C. BALPE ${ }^{2}$ \\ ${ }^{1}$ SATIE, ENS Cachan, 61, av President Wilson, F-94230 Cachan, France \\ ${ }^{2}$ HISPANO-SUIZA, Division SAFRAN POWER, Rond-point Rene-Ravaud, BP42, 77551 \\ Moissy-Cramayel, France
}

\begin{abstract}
This paper presents a 3-phase, 6-slot, and 4-pole Mutually Coupled Switched Reluctance Motor (MCSRM 6/4) with new current distribution. This kind of SRMs has both the merits of conventional SRMs and Fully Pitched SRMs, i.e. shorter end-windings and higher torque density. A comparison based on Finite Element Method (FEM) between conventional and mutually coupled SRMs, in terms of self flux-linkage and inductance per phase, mutual flux-linkage and inductance between phases, and output torque is realized. The conventional SRM is excited in unipolar mode, while the MCSRM is excited in bipolar overlapping mode. With a high coupling between phases for MCSRM, mutual inductances are employed to produce torque. Furthermore, the flux pathways are separated and distributed between phases, this leads to a less sensitivity to magnetic saturation. At high current density and high conduction angle, the MCSRM has a higher output torque and a lower torque ripple. Thus, comparing to conventional SRMs, the MCSRM is more outstanding for starter-generator applications (hybrid vehicles, aerospace) which needs high output torque.
\end{abstract}

Keywords-reluctance motor drive, Finite Element Method (FEM), mutually coupled switching reluctance machines

\section{Introduction}

$\mathrm{T}$ HANKS to their numerous advantages: high torque density, simplicity, robustness and low manufacturing cost, etc, the switched reluctance (SR) motors have attracted the electric machine researchers. However, the drawbacks of this kind of machines as high torque ripple, high acoustic noise and vibration limit their industrial applications [1], [2].

In most of the available literatures about the SRMs, the studied machines are conventional. The windings are wound around each tooth of stator, called short pitched winding SRMs (Fig. 1 (a)). This leads to a minimum magnetic coupling between phases and an easy electrical feeding. Thus, the torque can be simply derived from the rate of change of self inductance of the excited phase, and the flux of this phase is only a function of the phase current and the angular position of the rotor. For the SRMs excited in unipolar excitation mode, the positive torque is obtained by exciting the phase when its inductance rises [2]. Thus, each winding can only contribute to positive torque during a half of the electrical period.

In recent years, new winding configurations using the coupling between phases to produce higher torque density for 3-phase SRMs are studied in [3], [4]. Barrie C. Mecrow has presented a new winding configuration, which is a fun- damental departure from traditional machines, called Fully Pitched Winding SRMs. The torque is derived entirely from the rate of change of mutual inductance between phases instead of self inductance for conventional SRMs. With an appropriate excitation mode, the fully pitched windings SRMs can produce considerably higher density output torque. Whereas, comparing to the conventional SRMs with short pitched windings, fully pitched winding SRMs have longer end-winding and more copper loss at the same current density during one electric period. Furthermore, for the embedded systems which need small SRMs, the additional length due to the overlap of end-windings would be unacceptable.

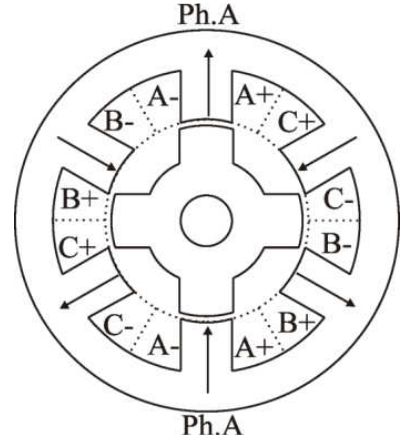

(a)

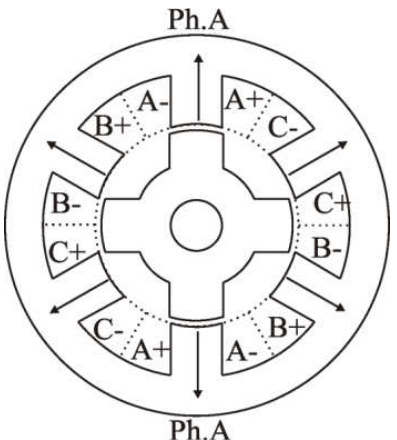

(b)
Fig. 1. The winding configurations of 3 phases, (a) conventional SRM 6/4, (b) MCSRM 6/4.

In order to overcome the drawbacks of fully pitched winding SRMs and keep their high output torque density performance, we present a new current distribution which has short pitched windings like conventional SRMs. Whereas, the torque is produced both from the rate of change of self inductances as the conventional short pitched winding SRMs and from the rate of change of mutual inductances between phases like the fully pitched winding SRMs. Furthermore, with the contribution of mutual inductances, this kind of machines could produce much higher output torque density. As in [5], we call them, Mutually Coupled Switched Reluctance Motors (MCSRMs). The current distribution is in Fig. 1 (b). To some degree, the machine with novel current distribution in this paper is a bridge between the conventional SRMs and the fully pitched SRMs.

A comparison between conventional and mutually coupled SRMs is included in terms of self flux-linkage, self inductance per phase, mutual flux-linkage, mutual inductances between 
phases, and output torque. The results based on Finite Element analysis (ANSYS) were presented.

II. Electromagnetic performance of the different SRMs

\section{A. Winding configurations and flux distribution}

In order to clarify the demonstration of winding configuration, a 3-phase, 6-slot, and 4-pole switched reluctance motor is chosen. Each tooth of either conventional or mutually coupled SRM carries a coil. In each slot, there are two windings of different phases. The problem of this configuration is that different phases are neither magnetically nor physically isolated. Thus, the fault tolerance is relatively lower than the fully pitched winding configuration. Whereas, due to the short pitched winding configuration, there is no overlap end-windings between phases. This leads to a shorter endwinding and lower copper losses at the same current density. For the conventional SRM in question, the current distributions of 3 phases is $\mathrm{A}+$ _A-_B-_B+_C+_C-_A _A+_B+_B-_C-_C+ (see Fig. 1(a)). As for mutually coupled SRMs, we change only the order of the winding in stator slots, i.e. A+_A-_B+_B-_C+_C-_A+_A-_B+_B-_C+_C- (see Fig. 1 (b)). The phase flux-linkage can be computed from the following expressions:

$$
\varphi_{\text {phase }}=N \iint \vec{B}_{\text {airgap }} d \vec{S}_{\text {tooth }}=N \int \vec{A} d \vec{L}
$$

Where $\varphi_{\text {phase }}$ is phase flux-linkage, $\vec{B}_{\text {airgap }}$ is air gap flux density, and $\vec{S}_{\text {tooth }}$ is tooth tip surface area, the magnetic vector potential $\vec{A}$ can be applied, $N$ is winding number per coil, and $L$ is the stack length.

In this paper, the flux leakage is neglected. Material (Fe-Co) properties defined as $\mathrm{BH}$ magnetizing curve are shown in Fig. 2.

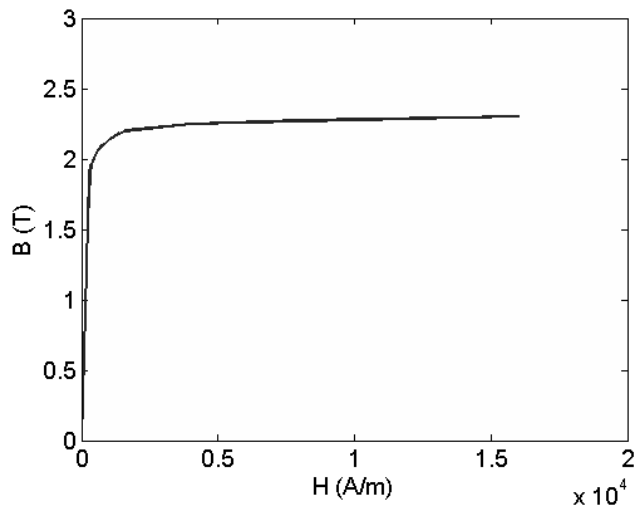

Fig. 2. Magnetizing curve of core materials

The typical flux distribution based on Finite Element (FE) at aligned position with the phase A excited in constant current is shown in the Fig. 3. The Fig. 3(a) shows that the flux of phase A passes approximately only through the stator back iron, the stator tooth of phase A, air gap, rotor poles, and rotor yoke. Thus, there is almost no mutual effect phase-to-phase. Consequently, the mutually coupled flux and in-ductance are negligible for conventional SRMs. Whereas, in Fig. 3(b), besides the same pathways like that of conventional SRM, the flux of phase A also passes through other phases, e.g. phase B and phase $\mathrm{C}$ for MCSRMs. At the aligned position, the mutual flux which passes through the phase $\mathrm{B}$ or phase $\mathrm{C}$ is about one half of the self flux of the phase A.

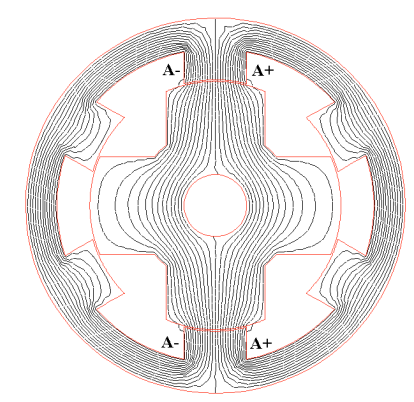

(a)

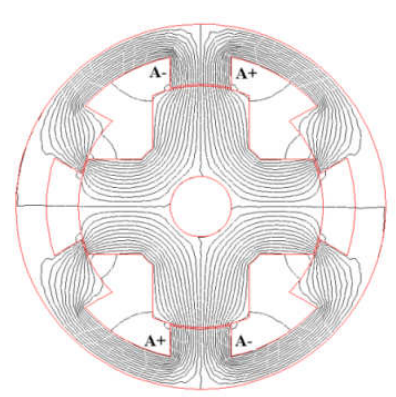

(b)
Fig. 3. Flux distribution with the phase A excited in constant current for 3-phase, 6-slot, and 4-pole SRMs, (a) conventional SRM, (b) mutually coupled SRM (MCSRM).

B. Self and mutual inductance

Considering the magnetic saturation effect, the finite element predicted self inductance of phase $A$ and mutual inductance between phase $\mathrm{A}$ and phase $\mathrm{B}$ at the phase $\mathrm{A}$ excited in constant current as shown in Fig. 4.

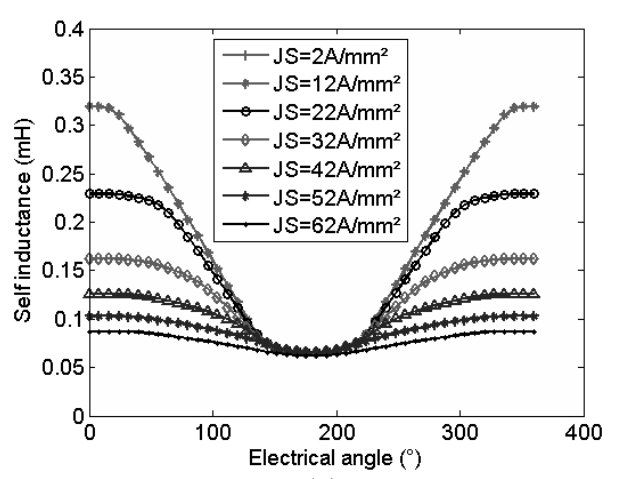

(a)

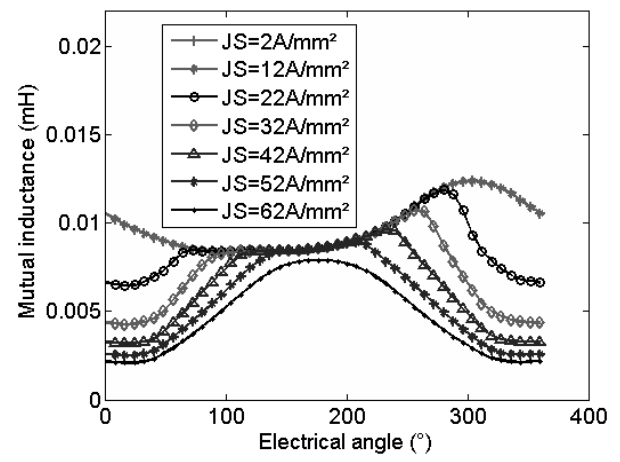

(b)

(i) 


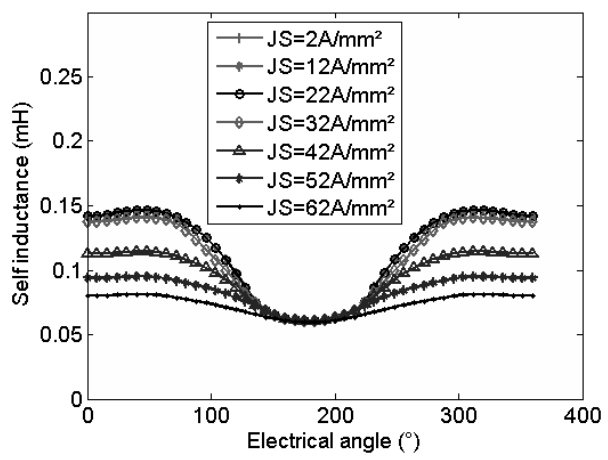

(a)

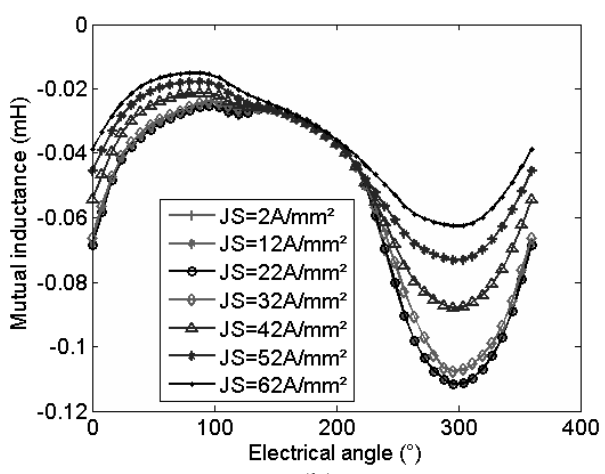

(b)

(ii)

Fig. 4. Self and mutual inductance versus rotor position during an electrical period, (i) conventional SRM, (a) self inductance, (b) mutual inductance; (ii) MCSRM, (a) self inductance, (b) mutual inductance (in this paper, the mutual inductance is between phase A and phase $\mathrm{B})$.

For the conventional SRM, self inductance per phase $(0.25 \mathrm{mH})$ is about 62 times higher than mutual inductance between phases $\left(4.10^{-3} \mathrm{mH}\right)$. As a consequence, mutual inductance for conventional SRM is negligible against self inductance and therefore is neglected. Whereas, neglecting magnetic saturation, self inductance of MCSRM is theoretically 2 times higher than mutual inductance between phases. With the saturation of machine, this ratio decreases to about 1.4 times. Thus, the mutual inductances between phases are not negligible, and which have an important role in the torque produce.

\section{Torque generation and torque ripple}

\section{A. Principle of torque production}

In the case of linear material, the expression of torque in conventional and mutually coupled SRMs is given as follows:

$$
\begin{aligned}
T= & \frac{1}{2} i_{a}^{2} \frac{d L_{a}}{d \theta}+\frac{1}{2} i_{b}^{2} \frac{d L_{b}}{d \theta}+\frac{1}{2} i_{c}^{2} \frac{d L_{c}}{d \theta} \\
& +i_{a} i_{b} \frac{d M_{a b}}{d \theta}+i_{a} i_{c} \frac{d M_{a c}}{d \theta}+i_{b} i_{c} \frac{d M_{b c}}{d \theta}
\end{aligned}
$$

Due to the fact that the mutual inductances between phases in conventional SRM are neglectable, and thus the last three terms of the torque expression are neglected. Contrarily, in the
Mutually Coupled SRMs, mutual inductances between phases have an important role in torque production, and thus should be taken into account for the torque computation. From (2), we found that, at the same phase current, the output torque is determined directly from the rate of change of inductances (for conventional SRM, self inductances; for MCSRM, self and mutual inductances). Thus, evaluating the variation of self and mutual inductances is necessary in this paper. For simplicity, we have developed an expression so as to present the variation of inductances, shown as follows:

$$
\begin{aligned}
& \Delta L=L_{\text {max }}-L_{\text {min }} \\
& \Delta M_{i j}=M_{i j_{\_} \max }-M_{i j_{-} \min } \\
& i, j=A, B, C, i \neq j
\end{aligned}
$$

Where $\Delta L$ and $\Delta M_{i j}$ are respectively variations of self and mutual inductance during an electrical period; $L_{\max }, L_{\text {min }}$, $M_{i j \_ \text {max }}$, and $M_{i j \_ \text {min }}$ are the maximum and minimum self inductance per phase, and maximum and minimum mutual inductance between phases during an electrical period, respectively. From expression (2) to (4), we assume that $\Delta \theta$ is the same and constant, we can state that the greater the value of $\Delta L$ or $\Delta M_{i j}$, the quicker the variation of inductances, and thus, the higher the output torque due to the concerned inductances. The results based on finite element analysis of variations of self and mutual inductances are shown in the Fig. 5.

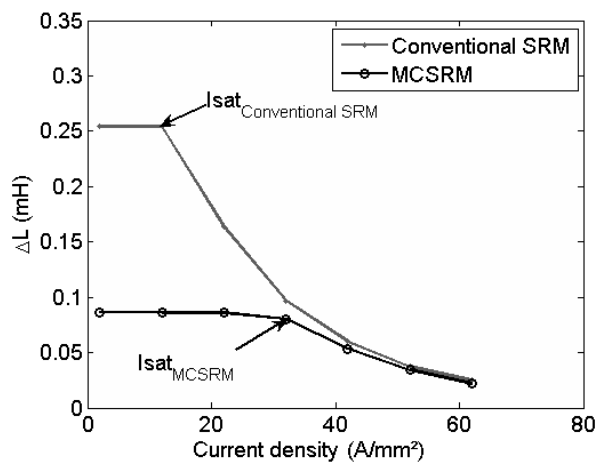

(a)

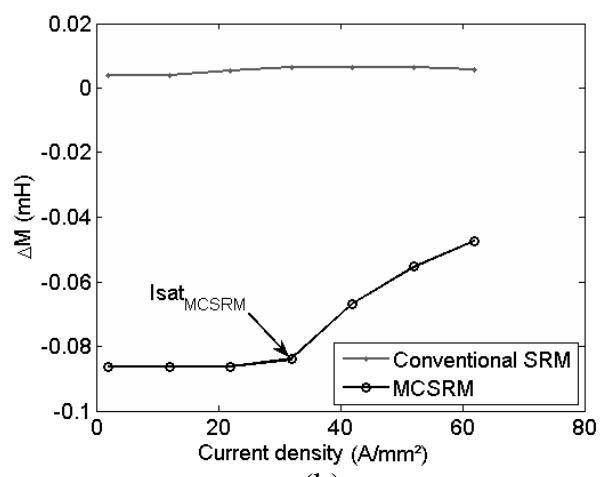

(b)

Fig. 5. Variation of self and mutual inductance versus current density, (a) self inductance per phase, (b) mutual inductance between phases (in this paper, the mutual inductance is between phase A and phase B). 
We found that, at low current density, e.g. $J S=5 \mathrm{~A} / \mathrm{mm}^{2}$, neither conventional nor MCSRM is saturated, because the currents of saturation of conventional and MCSRM

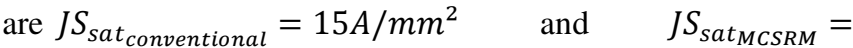
$33 \mathrm{~A} / \mathrm{mm}^{2}$, respectively. Neglecting the mutual inductances for conventional SRM, the variation of self inductance of conventional SRM is similar to the sum of variation of self and mutual inductance of MCSRM. This consequently leads to a similar average torque at low current density. With the increasing of current density, e.g. $J S=60 \mathrm{~A} / \mathrm{mm}^{2}$, self and mutual inductances of the conventional SRM become saturated. As a consequence, the rates of change of self and mutual inductance decrease. While the MCSRM is less sensitive to the magnetic saturation, and the sum of variation of self and mutual inductance decreases much less quickly than conventional SRM. Thus, the latter produce much lower average torque at high currents than the MCSRM.

\section{B. Excitation modes}

In this paper, we detailed two excitation modes, i.e. unipolar excitation mode for conventional SRM and bipolar excitation mode for MCSRM (Fig. 6) [2], [6], [7].

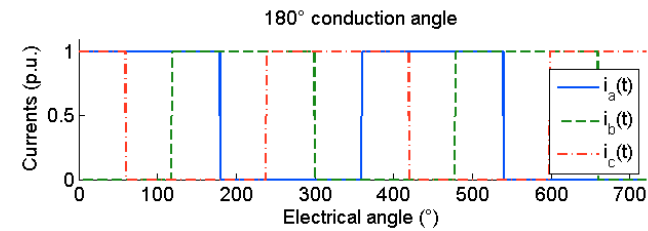

(a)

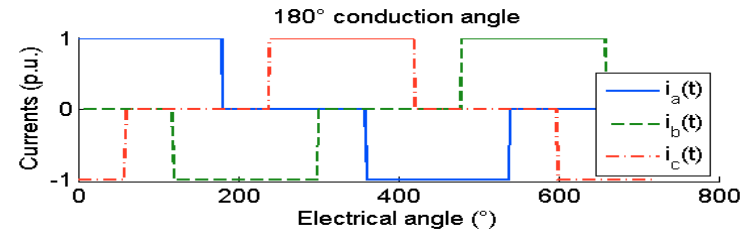

(b)

(i)

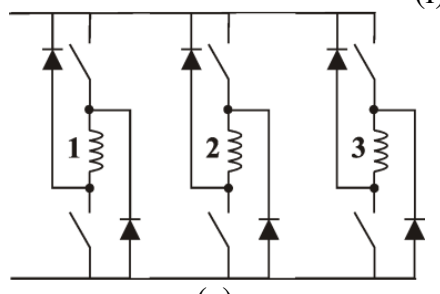

(a)

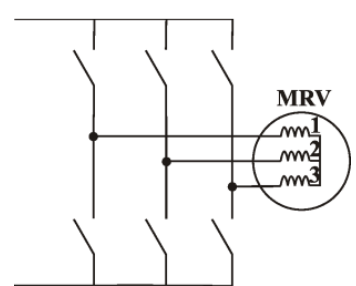

(b) (ii)

Fig. 6. Excitation modes and inverter circuits, (i) excitation modes, (ii) schematic of inverter for 3-phase; (a) conventional unipolar mode, (b) bipolar mode.

For conventional SRM, as in (2), the torque is directly proportional to $\partial L / \partial \theta$ and $I^{2}$, the latter is always positive regardless of the signs of excited current. Thus, the unipolar excitation mode (Fig. 6 (i) (ii) (a)) is employed for conventional SRM. For the mutually coupled SRM, the torque due to self inductance is positive as in conventional SRM, while $\partial M_{i j} / \partial \theta<0$. In order to obtain a positive torque, we have to make sure that $I_{i} I_{j}<0$. Thus, a conduction overlap between phases is needed and during the overlapping period, the concerned current signs should be opposite, i.e. $I_{i}<0$ and $I_{j}>0$ [8]. The bipolar excitation mode (Fig. 6 (i) (ii) (b)) is consequently employed. In order to study the influence of mutual inductances about torque production, a comparison of different conduction angles is realized. In this paper, the interval of conduction electrical angle is $\left[120^{\circ}, 180^{\circ}\right]$, thus the overlapping angle between phases is from $0^{\circ}$ to $60^{\circ}$. When the overlapping angle is $0^{\circ}$, although mutual inductances between phases of the MCSRM exist, there is no torque production due to mutual inductances, because $I_{i} I_{j}=0$. In this case, the resultant torque is only due to self inductances as for conventional SRM.

\section{Simulation based on Finite Element (FE)}

The simulation based on FE in terms of average versus current density is shown in Fig. 7.

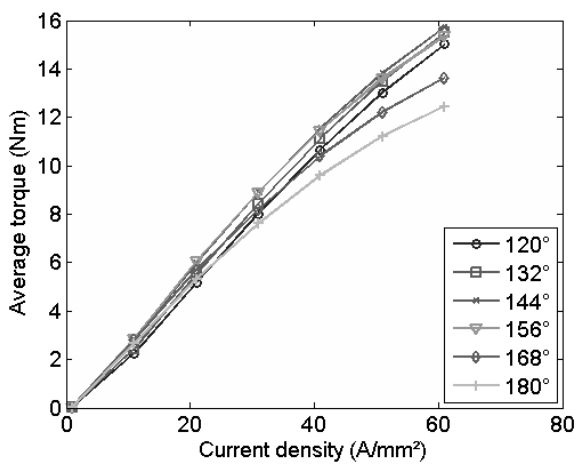

(a)

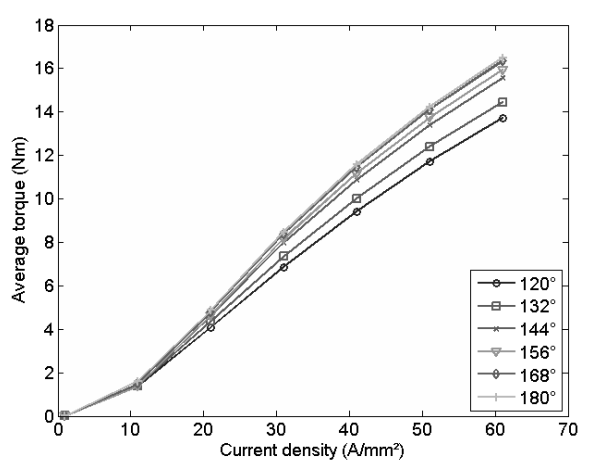

(b)

Fig. 7. Comparison of output torque versus current density for different conduction angles, (a) conventional SRM, (b) MCSRM

For conventional SRM (Fig. 7 (a)), at low current density, the average torque increases with the increase of conduction angles. Whereas, at high current density, the machine begins to saturate, further, the higher conduction overlap between phases makes the machine more saturated. As consequence, the rate of change of self inductance decreases, the same as the average torque. For the MCSRM, the average torque increases with the increase of conduction angles regardless of current density until the machine is strongly saturated. Finally, at high current density $\left(J S=60 \mathrm{~A} / \mathrm{mm}^{2}\right)$ and high conduction angle $\left(180^{\circ}\right)$, the MCSRM produces much higher average torque 
$(16.5 \mathrm{Nm})$ than that of conventional SRM $(12.4 \mathrm{Nm})$, while in this case, the copper losses of the two machines are the same, because the conduction angle, the current density, and the volume of copper in stator slots are the same.

For industrial applications, the torque ripple is an important electromagnetic performance parameter. To some degree, a very high torque ripple could limit the machine's application although the output torque is more important at the same current. Thus, the comparison of the torque ripple between conventional and mutually coupled SRMs is necessary. The torque ripple coefficient is calculated as follows:

$$
\Delta T=\left(T_{\max }-T_{\min }\right) / T_{a v}
$$

Where $T_{\max }, T_{\min }$, and $T_{a v}$ are respectively the maximum, the minimum, and the average torque during an electrical period. The finite element results are shown in Fig. 8. We found that, for conventional SRM (Fig. 8 (a)), at low current and low conduction angles, e.g. $120^{\circ}-132^{\circ}$, the torque ripple decreases with the increase of current density, while at high conduction angles, e.g. $168^{\circ}-180^{\circ}$, the torque ripple increases with the increase of current density. For MCSRM (Fig. 8 (b)), the torque ripple decreases with the increase of current density regardless of the conduction angles until the machine is strongly saturated. However, at high current density, e.g.J $S=$ $60 \mathrm{~A} / \mathrm{mm}^{2}$, and high conduction angles $\left(180^{\circ}\right)$, the MCSRM has a much lower torque ripple coefficient (0.6) than conventional SRM (1.5). This makes the MCSRM more outstanding at high current density and high conduction angles.

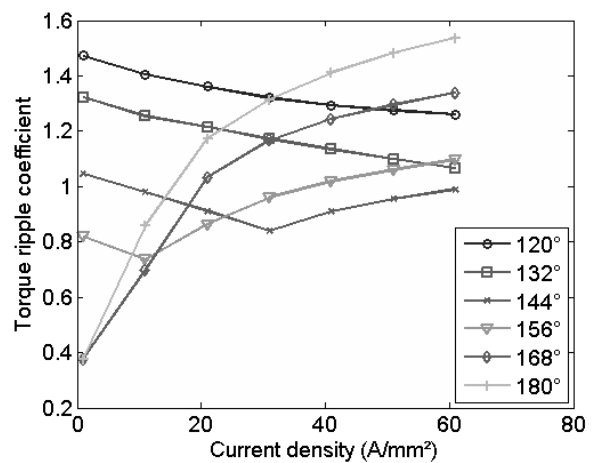

(a)

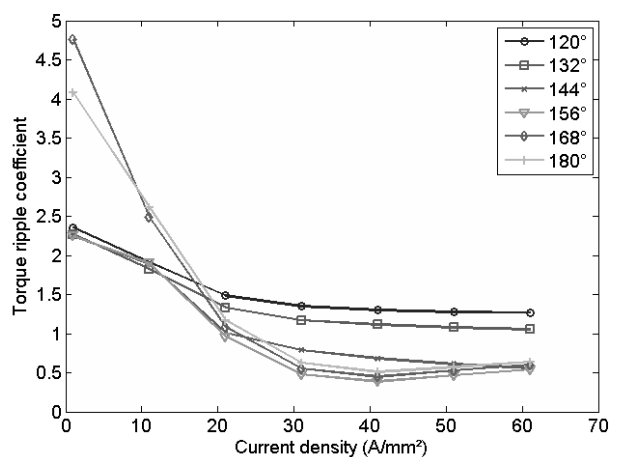

(b)

Fig. 8. Torque ripple coefficient versus current density for conventional and MCSRM
After previous studies, it is interesting to increase the conduction angle for MCSRM in order to utilize enough the mutual inductance and obtain higher torque density. To archive this aim, we can take away the zero-current period in (Fig. 6 (i) (b)). As for the Permanent Magnet (PM) Brushless Machines, the Brushless DC (rectangular current) or the Brushless AC (sinusoidal current) control strategies can be employed. The current waveforms are shown in Fig. 9. Comparing to the half-H-bridge control strategy for conventional SRM (Fig. 6 (ii) (a)), the 6 diodes are taken away (Fig. 6 (ii) (b)), the control strategy is much simpler, and the cost of the machine is dramatically decreased.

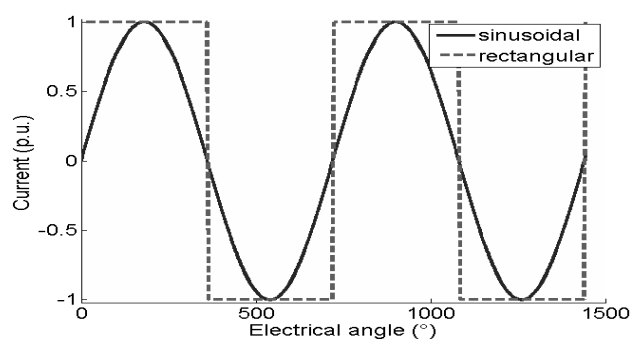

Fig. 9. Current waveforms for BLDC and BLAC control strategies

The output torque versus current density for different current waveforms is shown in Fig. 10.

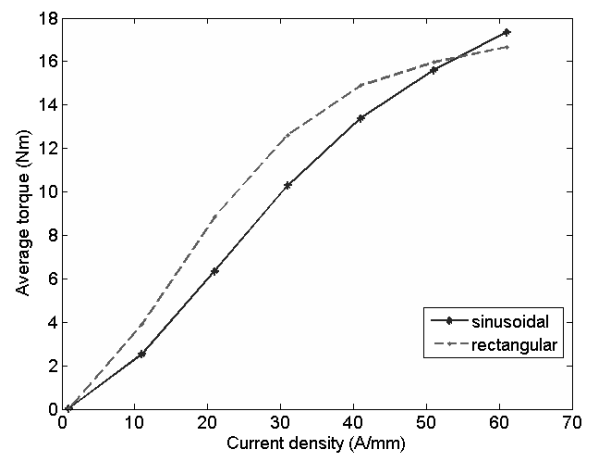

(a)

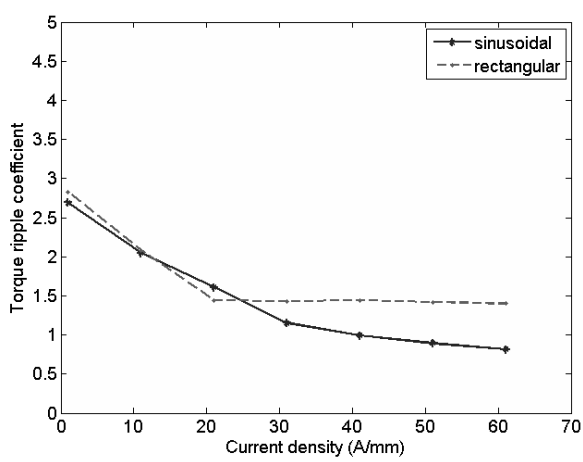

(b)

Fig. 10. Comparison of output torque and torque ripple coefficient versus current density for different current waveforms, (a) output torque, (b) torque ripple coefficient.

We find that the MCSRM with rectangular current excitation can produce higher output torque than the MCSRM with sinusoidal current excitation. While at higher current density, the torque ripple due to sinusoidal current control is 
lower than that of rectangular control. Nevertheless, MCSRM with these two control strategies, which have a simpler inverter circuit, can produce higher output torque and similar torque ripple comparing to the previous control strategies.

\section{Conclusion}

The comparison of performances between the 3-phase, 6slot, 4-poles conventional and mutually coupled SRMs has been presented in this paper. It has shown that different kinds of current distributions and different excitation modes have an important influence over the electromagnetic performances of SRMs. For the mutually coupled SRM, the flux pathways are separated and distributed between phases, thus, the contacted surface areas between stator teeth tips and the air gap increase. This leads that the MCSRM is less sensitive to the magnetic saturation than conventional SRM. At high current density and high conduction angles, the conventional SRM is saturated, its rates of change of self and mutual inductances decrease, while the rate of change of self and mutual inductances of MCSRM decreases considerably less quickly. Consequently, at high current density and high conduction angles, the average torque of the MCSRM is higher while the torque ripple coefficient is much lower than the conventional SRM. This makes MCSRM an outstanding candidate for the starter-generator application (aerospace or Hybrid vehicle) [9], [10]. Moreover, the inverter circuit of MCSRM has only 6 transistors, while, the inverter circuit of conventional SRM, which has 6 transistors and 6 diodes. This makes the MCSRM inverter circuit much simpler and less expensive.

\section{References}

[1] X. Ojeda, X. Mininger, M. Gabsi, M. Lecrivain, "Sinusoidal Feeding for Switched Reluctance Machine: Application to Vibration Damping", ICEM 2008, Vilamoura, Portugal, September 2008.

[2] Z. Q. Zhu and D. Howe, "Electrical Machines and Drives for Electric, Hybrid, and Fuel Cell Vehicles,', Proc. IEEE, vol. 95, no. 4, pp. 746765, Apr. 2007.

[3] B. C. Mecrow, "New winding configuration for doubly salient Reluctance machines," IEEE Trans. Ind. Applicat., vol. 32, pp. 13481356, Nov./Dec. 1996.

[4] B. C. Mecrow, C. Weiner, A. C. Clothier, "The Modeling of Switched Reluctance Machines with Magnetically Coupled Winding," IEEE Trans. Ind. Applicat., vol. 37, no.6, pp. 1675-1683, Nov./Dec. 2001.

[5] J. M. Kokernak, and D. A. Torrey, "Magnetic Circuit Model for the Mutually Coupled Switched-Reluctance Machine,', IEEE Trans. On Magnetics, vol. 36, no. 2, Mar. 2000, pp. 500-507

[6] J. M. Miller, A. R. Gale, P. J. McCleer, F. Leonardi, and J. H. Lang, "Starter/alternator for hybrid electric vehicle: Comparison of induction and variable reluctance machines and drives," in Proc. IEEE 1998 Industry Applications Soc. Annu. Meeting, Oct. 12-15, 1998, pp. 513 523.

[7] C. S. Edrington, M. Krishnamurthy, and B. Fahimi, "Bipolar switched reluctance machines: A novel solution for automotive applications,' IEEE Trans. Veh. Technol., vol. 54, no. 3, pp. 795-808, May 2005.

[8] D. Panda, V. ramanarayanan, "Mutual Coupling and Its Effect on Steady-State Performance and Position Estimation of Even and Odd Number Phase Switched Reluctance Motor Drive"' IEEE Trans. On Magnetics, vol. 43, no. 8, Aug. 2007, pp. 3445-3456

[9] B. Fahimi, A. Emadi, and R. B. Sepe, "A switched reluctance machine-based starter/alternator for more-electric cars," IEEE Trans. Energy Convers., vol. 19, no. 1, pp. 116-124, Mar. 2004.

[10] S. H. Wang Q. H. Zhan Z. Y. Ma L. B. Zhou, "Implementation of a 50-kW Four-Phase Switched Reluctance Motor Drive System for Hybrid Electric Vehicle,', IEEE Trans. On Magnetics, vol. 41, no. 1, Jan. 2005, pp. 501-504

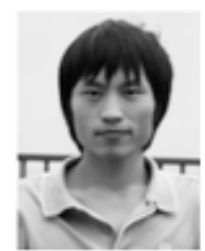

Guangjin LI was born in Xiaogan, China, in 1984. He received his BS and MSc degree in Electrical Engineering from the University of Wuhan, China, in 2007 and Paris XI, Paris, France, in 2008, respectively. He is currently a Ph.D student in the laboratory SATIE of the Ecole Normale Supérieure (ENS) de Cachan, Paris, France. His main research interests include the design and the optimization of the Switched Reluctance Machines (SRMs) and the Flux-Switching Permanent Magnet Machines (FSPMMs). The available contact of the author is: guangjin.li@satie.enscachan.fr

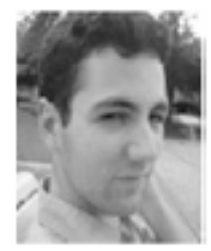

Xavier Ojeda was born in 1980 in Buenos Aires, Argentina. He received the BS and the MSc degrees in electrical engineering from the Ecole Nor-male Supérieure de Cachan (ENS Cachan, France) and Paris$\mathrm{XI}$ University. $\mathrm{He}$ is actually preparing his $\mathrm{Ph} . \mathrm{D}$ in Electrical Engineering in SATIE, Systèmes et Applications des Technologies de l'Information et de l'Energie (ENS Cachan, France), laboratory. His main research topics are active damping of SRM motors for high speed applications and machines noise modeling.

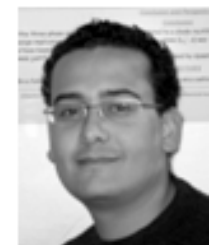

Sami Hlioui was born in Sfax, Tunisia, in October 20, 1980. He received the M.Sc. degree in electrical engineering from the Ecole Normale Supérieure de Cachan, Cachan, France and the Ph.D. degree in electrical power engineering from the University of Technology of Belfort-Montbeliard (UTBM) France in 2008. He is now postdoctoral researcher in the SATIE Laboratory of the "Ecole Normale Supérieure de

Cachan"Cachan, France.His interests include multidisciplinary design of electrical machines.

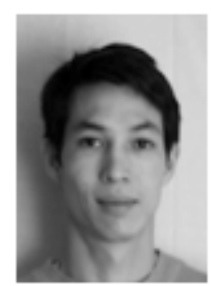

Emmanuel Hoang was born in Antibes, France, in 1966. He receive the "agrégation" in electrical engineering in 1990 and the Ph.D degrees from the Ecole Normale Supérieure de Cachan, in 1995. Since 1990, he works with the electrical machine team in the SATIE laboratory. His research interests the modeling of the iron losses in SRMs and the design, modeling, optimization and control of novel topologies of PM machines. hoang@satie.ens-cachan.fr

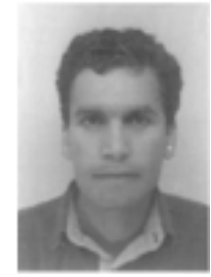

Mohamed Gabsi received his Ph.D degree in electrical engineering from University of Paris-VI in 1987 and his HDR in 1999 from University of Paris-XI (Orsay, France). Since 1990, he is working in electrical machine team (SETE, Systèmes d' Energies pour le Transport et l'Environnement) of SATIE laboratory where he is currently a professor and researcher. His research interests include SRM, vibrations and acoustic noise and PM machines.

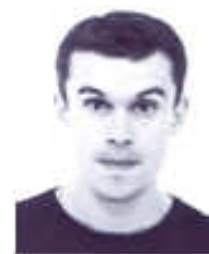

Cedric BALPE was born in 1978 in Carhaix, France. $\mathrm{He}$ received $\mathrm{MSc}$ degree in electrical engineering from the Ecole Polytechnique de Nantes, Electrical department located in Saint-Nazaire, France. Since 2008, he been working for the SAFRAN POWER Division of HISPANO-SUIZA Company located in Reau, France. He is involved in costumers' requests for quotation and products architecture definition.

$\mathrm{He}$ is also in charge of advanced studies and partnerships with French laboratories. His interests include high temperature systems (electrical motors design, power electronics...) and electrical motors control. 